\author{
LINEAR AND NON-LINEAR THEORY OF \\ GENERALIZED FUNCTIONS AND ITS APPLICATIONS \\ BANACH CENTER PUBLICATIONS, VOLUME 88 \\ INSTITUTE OF MATHEMATICS \\ POLISH ACADEMY OF SCIENCES \\ WARSZAWA 2010
}

\title{
THE STRUCTURE OF QUASIASYMPTOTICS OF SCHWARTZ DISTRIBUTIONS
}

\author{
JASSON VINDAS \\ Department of Mathematics, Louisiana State University \\ Baton Rouge, LA 70803, U.S.A. \\ Current address: Department of Pure Mathematics and Computer Algebra, Ghent University \\ Krijgslaan 281 Gebouw S22, B 9000 Gent, Belgium \\ E-mail:jvindas@cage.ugent.be
}

\begin{abstract}
In this article complete characterizations of the quasiasymptotic behavior of Schwartz distributions are presented by means of structural theorems. The cases at infinity and the origin are both analyzed. Special attention is paid to quasiasymptotics of degree -1 . It is shown how the structural theorem can be used to study Cesàro and Abel summability of trigonometric series and integrals. Further properties of quasiasymptotics at infinity are discussed. A condition for test functions in bigger spaces than $\mathcal{S}$ is presented which allows one to consider the respective quasiasymptotics over them. An extension of the structural theorems for quasiasymptotics is given. The author studies a structural characterization of the behavior $f(\lambda x)=O(\rho(\lambda))$ in $\mathcal{D}^{\prime}$, where $\rho$ is a regularly varying function.
\end{abstract}

1. Introduction. The concept of the quasiasymptotic behavior of distributions was introduced by B. I. Zavialov for tempered distributions in [29] and was studied comprehensively in [24]. Later this concept was slightly reformulated in [11, 12].

The quasiasymptotics of distributions have shown to be of importance in several areas such as mathematical physics [24, 29], abelian and tauberian theory of integral transforms $[16,24,25]$, asymptotic behavior of solutions of partial differential equations $[5,8,21,24$, $28]$, and summability of trigonometric series and integrals $[6,8,21,22,26,27]$.

Since its introduction, the study of the structure of the quasiasymptotics has deserved a special place $[5,6,10-16,19,22,24]$. S. Łojasiewicz introduced the value of a distribution at a point, and he provided the corresponding structural theorem for it. V. S. Vladimirov, Yu. N. Drozhzhinov and B. I. Zavialov gave a complete structural theorem for quasiasymptotics at infinity of tempered distributions with support on cones.

2000 Mathematics Subject Classification: Primary 46F10, 41A60; Secondary 26A12, 42 A24. Key words and phrases: quasiasymptotic behavior, asymptotic behaviors of distributions. The paper is in final form and no version of it will be published elsewhere. 
S. Pilipović gave partial structural theorems for one dimensional quasiasymptotics at the origin and infinity. However, a complete structural theorem for quasiasymptotics has been an open question for long time.

The purpose of this note is to report a solution for this open question in the one dimensional case and discuss some of its consequences and generalizations. A complete structural theorem for quasiasymptotics at the origin has been recently obtained by the author and S. Pilipović in [23]. The case at infinity has been studied by the author in [20]. In this paper we summarize these new results. The proofs of the structural theorems will be omitted since the details can be found in cited works [20,23]. Therefore, the author concentrates on studying some consequences and giving some extensions of them.

The plan of this article is as follows. In Section 2, the structural theorems will be stated. We will then discuss in Section 3 a particular case of the structural theorems within the context of summability of Fourier series and integrals. Actually, it should be mentioned that many of the ideas to study the structure of quasiasymptotics, especially in the case of degrees in $\mathbb{Z}_{-}$, were inspired by techniques previously applied by the author and R. Estrada in $[6,21,22]$ at studying the value of a distribution at a point and distributional jump behaviors in connection with trigonometric series and integrals. In Section 4, the structure of quasiasymptotics at infinity is applied to show that the quasiasymptotic behavior holds in smaller spaces than $\mathcal{S}^{\prime}$, namely in some spaces of the type $\mathcal{K}_{\beta}^{\prime}$. In Section 5 , a generalization of the structural theorems for quasiasymptotics is given, this is generalized to quasiasymptotically bounded distributions. The article is ended by posing two important open questions concerning the quasiasymptotic behavior of distributions, it is done in Section 6.

2. The structure of quasiasymptotics. The purpose of this section is to present the structural theorems for quasiasymptotic behaviors of distributions. We first fix the notation to be used and introduce the basic definitions. All of our spaces of functions and distributions will be taken over the real line. The Schwartz spaces of test functions and distributions over the real line are denoted by $\mathcal{D}$ and $\mathcal{D}^{\prime}$, respectively. The spaces of smooth rapidly decreasing functions and its dual, the space of tempered distributions, are denoted by $\mathcal{S}$ and $\mathcal{S}^{\prime}$. The spaces $\mathcal{E}$ and $\mathcal{E}^{\prime}$ are the test space of all $\mathcal{C}^{\infty}$-functions and its dual, the space of compactly supported distributions. The spaces $\mathcal{D}_{+}^{\prime}$ and $\mathcal{S}_{+}^{\prime}$ denote the subspaces of $\mathcal{D}^{\prime}$ and $\mathcal{S}^{\prime}$, respectively, consisting of the distributions and tempered distributions with support in $[0, \infty)$. We refer the reader to $[9,17,24]$ for properties of these spaces.

The main subject of this section is the so-called quasiasymptotic behaviors of distributions at infinity and the origin which we now proceed to define $[9,11,12,16,24,25$, 29]. There are several definitions in the literature. Let us start with the one from [11, 12]. Let $\rho$ be a positive measurable function defined in some neighborhood of infinity (respectively of the origin), we say that $f \in \mathcal{D}^{\prime}$ has quasiasymptotic behavior at infinity in $\mathcal{D}^{\prime}$ (respectively at the origin in $\mathcal{D}^{\prime}$ ) if for some $g \in \mathcal{D}^{\prime}, g \neq 0$,

$$
\lim _{\lambda \rightarrow \infty}\left\langle\frac{f(\lambda x)}{\rho(\lambda)}, \phi(x)\right\rangle=\langle g(x), \phi(x)\rangle, \quad \phi \in \mathcal{D},
$$


(respectively in the case at the origin the limit is taken as $\lambda \rightarrow 0^{+}$). In [24] the original definition for the quasiasymptotic behavior at infinity is given only for $f \in \mathcal{S}_{+}^{\prime}$ and hence the test function $\phi$ is allowed to be in $\mathcal{S}$; there the function $\rho$ is called an automodel function but we will not follow this terminology. In [11-14], the definition is extended to the form just presented here. It follows from the definition that $\rho$ and $g$ in (1) cannot have an arbitrary form $[9,16,24]$. Indeed, $\rho$ must be a regularly varying function $[2,18]$ and $g$ must be a homogeneous distribution [9] having degree of homogeneity equal to the index of regular variation of $\rho$.

We briefly recall the concepts of regularly varying functions and homogeneous distributions, in this way the notation will be fixed.

A real-valued measurable function $\rho$ defined in some interval of the form $[A, \infty)$, $A>0$, is called regularly varying at infinity if $\rho$ is positive near $\infty$ and there exists an $\alpha \in \mathbb{R}$, called the index of regular variation of $\rho$, such that

$$
\lim _{\lambda \rightarrow \infty} \frac{\rho(a \lambda)}{\rho(\lambda)}=a^{\alpha}
$$

for any $a>0$. If $\alpha=0$, then the function is called slowly varying at infinity; the letter $L$ is commonly used for denoting slowly varying functions, we should follow this convention in this article. Note that $\rho$ is regularly varying if and only if it can be written as $\rho(\lambda)=\lambda^{\alpha} L(\lambda)$, where $L$ is slowly varying. In the same way one defines regularly and slowly varying functions at the origin. We refer to $[2,18]$ for properties of such functions.

Since we are dealing with the one dimensional case, we know explicitly all the homogeneous distributions on the real line [9, p. 72]. One has that either a homogeneous distribution has the form

$$
g(x)=C_{-} x_{-}^{\alpha}+C_{+} x_{+}^{\alpha}, \quad \text { if } \alpha \notin\{-1,-2,-3, \ldots\},
$$

for some constants $C_{-}$and $C_{+}$, or

$$
g(x)=\gamma \delta^{(k-1)}(x)+\beta x^{-k}, \quad \text { if } \alpha=-k \in\{-1,-2,-3, \ldots\},
$$

for some constants $\gamma$ and $\beta$, where here we are following the notation from [9]. When $k=1$, we sometimes denote the distribution $x^{-1}$ by p.v. $(1 / x)$. Other special distributions we will frequently use are the Heaviside function $H$, i.e., the characteristic function of $(0, \infty)$, and the pseudofunctions $\operatorname{Pf}(H(x) / x)$ and $\operatorname{Pf}(H(-x) / x)$, also defined in [9].

As mentioned before, in the usual definition of the quasiasymptotic behavior the distribution $g$ is assumed to be different from 0 , however in $[20,23]$ this definition is extended by allowing $g$ to be 0 , this is done because the results about the structure of the quasiasymptotics are also valid in this case. In order to introduce some language, we state this definition.

Definition 2.1. Let $L$ be slowly varying at infinity (respectively at the origin). We say that $f \in \mathcal{D}^{\prime}$ has quasiasymptotic behavior at infinity in $\mathcal{D}^{\prime}$ with respect to $\lambda^{\alpha} L(\lambda)$ (respectively at the origin), $\alpha \in \mathbb{R}$, if for some $g \in \mathcal{D}^{\prime}$,

$$
\lim _{\lambda \rightarrow \infty}\left\langle\frac{f(\lambda x)}{\lambda^{\alpha} L(\lambda)}, \phi(x)\right\rangle=\langle g(x), \phi(x)\rangle, \quad \phi \in \mathcal{D},
$$


(in the case at the origin $\lambda \rightarrow 0^{+}$). If (2) holds, it is also said that $f$ has quasiasymptotics of degree $\alpha$ at infinity (the origin) with respect to the slowly varying function $L$.

We also express $(2)$ by using the asymptotic notation

$$
f(\lambda x)=\lambda^{\alpha} L(\lambda) g(x)+o\left(\lambda^{\alpha} L(\lambda)\right) \quad \text { as } \lambda \rightarrow \infty \text { in } \mathcal{D}^{\prime},
$$

which should always be interpreted in the weak topology of $\mathcal{D}^{\prime}$, i.e., in the sense of (2). Sometimes one could have quasiasymptotics in other spaces of distributions. For instance, if $\mathcal{A}$ and $\mathcal{A}^{\prime}$ are spaces of test functions and distributions, one writes $\mathcal{A}^{\prime}$ instead of $\mathcal{D}^{\prime}$ in (3) if $f \in \mathcal{A}^{\prime}$ and (2) holds for all $\phi \in \mathcal{A}$.

We now want to make some comments about the previous known properties of the structure of the quasiasymptotics available in the literature. This is valuable for the reader since many important techniques can be found in the references.

We start with quasiasymptotics at infinity. The complete structural theorems for distributions from $\mathcal{S}_{+}^{\prime}$ can be found in [24]. In addition, in page 134 of the cited book, one finds a decomposition theorem, which basically implies the structural theorem when the degree of the quasiasymptotic behavior is not a negative integer with no restriction on the support of the distribution. The details about how this is implied by the decomposition theorem can be found in [11]. Therefore, in the case at infinity the only unknown structural theorem was for quasiasymptotics whose degrees are negative integers. In a recent paper [22] a structural theorem for the quasiasymptotic behavior of degree -1 with respect to the trivial slowly varying function, $L \equiv 1$, was obtained. The technique employed was based on the concept of asymptotically homogeneous functions of degree 0 with respect to the trivial slowly varying function, previously used in [6] to characterize Łojasiewicz point values of periodic distributions. In the case at the origin, only partial results were known under restrictions on the degree of the quasiasymptotic and boundedness of $L$ [14]. The reader can also consult $[15,16,19]$ for more about these structural results.

We now proceed to state the structural theorems. The details for the proofs will not be supplied, they can be found in [20,23], we just mention that the proofs were based on the usage of asymptotically and associated asymptotically homogeneous functions. Since they are involved in the statements of the structural theorems, we give the definitions. The reader can find the main properties of such functions in the cited articles.

Definition 2.2. A function $b$ is said to be asymptotically homogeneous of degree $\alpha$ at infinity with respect to the slowly varying function $L$, if it is measurable and defined in some interval $[A, \infty), A>0$, and for each $a>0$,

$$
b(a \lambda)=a^{\alpha} b(\lambda)+o(L(\lambda)), \quad \lambda \rightarrow \infty .
$$

Definition 2.3. A function $b$ is said to be associate asymptotically homogeneous of degree 0 at infinity with respect to the slowly varying function $L$, if it is measurable and defined in some interval $[A, \infty), A>0$, and there is a constant $\beta$ such that

$$
b(a \lambda)=b(\lambda)+\beta \log a L(\lambda)+o(L(\lambda)), \quad \lambda \rightarrow \infty,
$$

for each $a>0$. 
REMARK 2.4. Related classes of functions having regular variation at infinity were introduced by R. Bojanić and J. Karamata in [4], these classes include associate asymptotically homogeneous functions. They are also studied in $[1,2,18]$.

With the obvious modifications one defines asymptotically and associate asymptotically homogeneous functions at the origin.

The structure of the quasiasymptotics when the degree is not a negative integer is described by the following theorem.

THEOREM 2.5. Let $f \in \mathcal{D}^{\prime}$ have the quasiasymptotic behavior at infinity (at the origin) in $\mathcal{D}^{\prime}$,

$$
f(\lambda x)=C_{-} L(\lambda) \frac{(\lambda x)_{-}^{\alpha}}{\Gamma(\alpha+1)}+C_{+} L(\lambda) \frac{(\lambda x)_{+}^{\alpha}}{\Gamma(\alpha+1)}+o\left(\lambda^{\alpha} L(\lambda)\right),
$$

$\lambda \rightarrow \infty$ (respectively $\lambda \rightarrow 0^{+}$). If $\alpha \notin \mathbb{Z}_{-}$, then there exist an integer $m \in \mathbb{N}, m+\alpha>0$, an m-primitive $F$ of $f$, i.e., $F^{(m)}=f$, such that $F$ is continuous (respectively continuous on a neighborhood of 0 ) and

$$
\lim _{x \rightarrow \pm \infty} \frac{\Gamma(\alpha+m+1) F(x)}{x^{m}|x|^{\alpha} L(|x|)}=C_{ \pm}
$$

(at the origin $x \rightarrow 0^{ \pm}$). Conversely, if these conditions hold, then (4) follows.

REMARK 2.6. Theorem 2.5 in the case of quasiasymptotics at the origin admits an interesting generalization whose proof is exactly the same as in [23]. The generalization is as follows. Suppose that $f$ is a distribution just defined on $\mathbb{R} \backslash\{0\}$ and $(2)$ holds just for $\phi \in \mathcal{D}\left(\mathbb{R} \backslash\{0\}\right.$ ) (here $\lambda \rightarrow 0^{+}$). If we assume that $\alpha \notin \mathbb{Z}_{-}$, then there are an $m \in \mathbb{N}$, $m+\alpha>0$, and a distribution $F$, which is a continuous function on some neighborhood of the origin, satisfying (5) (with the limit taken as $x \rightarrow 0^{ \pm}$), such that $F^{(m)}=f$ on $\mathbb{R} \backslash\{0\}$. Consequently, by taking $F^{(m)}$, one obtains an extension of $f$ to $\mathbb{R}$ which has quasiasymptotic behavior at the origin. On the other hand if $f$ is already defined on $\mathbb{R}$, then $f_{0}=F^{(m)}$ has quasiasymptotic behavior in $\mathcal{D}^{\prime}$ and

$$
f(x)=f_{0}(x)+\sum_{j=0}^{n} a_{j} \delta^{(j)}(x),
$$

for some constants $a_{0}, a_{1}, \ldots, a_{n}$. It should be noticed that the results of this remark extend the properties obtained by S. Eojasiewicz in [10] about the limit of a distribution at a point.

The case when $\alpha \in \mathbb{Z}_{-}$is somehow more complicated, the complete information is presented in the next theorem. We denote by $l_{m}$ the $m$-primitive of $\log |x|$ having the property that $l_{m}^{(j)}(0)=0$ for $0 \leq j<m$.

THEOREM 2.7. Let $f \in \mathcal{D}^{\prime}$ and let $k$ be a positive integer. Then $f$ has the quasiasymptotic behavior at infinity (at the origin) in $\mathcal{D}^{\prime}$,

$$
f(\lambda x)=\gamma L(\lambda) \delta^{(k-1)}(\lambda x)+(-1)^{k-1}(k-1) ! \beta L(\lambda)(\lambda x)^{-k}+o\left(\lambda^{-k} L(\lambda)\right),
$$

$\lambda \rightarrow \infty$ (respectively $\lambda \rightarrow 0^{+}$) if and only if there exist an $m \in \mathbb{N}, m>k$, a function $b$ defined on $(0, \infty)$, being associate asymptotically homogeneous of degree 0 at infinity (at 
the origin) with respect to $L$ and satisfying $b(a \lambda)=b(\lambda)+\beta \log a L(\lambda)+o(L(\lambda))$, and $a$ continuous $m$-primitive $F$ of $f$ (respectively continuous near 0 ) such that

$$
\begin{aligned}
F(x)=\frac{x^{m-k} b(|x|)}{(m-k) !}+\gamma \operatorname{sgn} x \frac{x^{m-k} L(|x|)}{2(m-k) !} & -\beta \frac{x^{m-k} L(|x|)}{(m-k) !} \sum_{j=1}^{m-k} \frac{1}{j} \\
& +o\left(|x|^{m-k} L(|x|)\right)
\end{aligned}
$$

as $x \rightarrow \pm \infty\left(x \rightarrow 0^{ \pm}\right)$in the ordinary sense. The last property is equivalent to

$$
\lim _{x \rightarrow \infty} \frac{(m-k) !\left(a^{k-m} F(a x)-(-1)^{m-k} F(-x)\right)}{x^{m-k} L(x)}=\gamma+\beta \log a,
$$

for each $a>0$ (respectively $\lim _{x \rightarrow 0^{+}}$). Moreover, (6) implies that

$$
\begin{gathered}
F(\lambda x)=b(\lambda) \frac{(\lambda x)^{m-k}}{(m-k) !}+\gamma L(\lambda) \frac{(\lambda x)^{m-k}}{2(m-k) !} \operatorname{sgn} x \\
+\beta L(\lambda) \lambda^{m-k} l_{m-k}(x) \\
+o\left(\lambda^{m-k} L(\lambda)\right)
\end{gathered}
$$

as $\lambda \rightarrow \infty$ (respectively $\left.\lambda \rightarrow 0^{+}\right)$, in the sense of convergence in $\mathcal{D}^{\prime}$.

REMARK 2.8. Even if initially not assumed, relation (7) holds uniformly for $a$ on compact subsets of $(0, \infty)$.

It should be noticed that in (7) it is not absolutely necessary to assume that the limit is of the form $\gamma+\beta \log a$ (however, (7) will force it to have this form). Indeed, we have the following corollary.

TheOREM 2.9. Let $f \in \mathcal{D}^{\prime}$. Then $f$ has quasiasymptotics at infinity (at the origin) of degree $-k, k \in \mathbb{Z}_{+}$, if and only if there exist a positive integer $m>k$ and a continuous (continuous near 0) m-primitive $F$ of $f$ such that for each $a>0$ the following limit exists

$$
\lim _{x \rightarrow \infty} \frac{a^{k-m} F(a x)-(-1)^{m-k} F(-x)}{x^{m-k} L(x)},
$$

(respectively the limit taken as $x \rightarrow 0^{+}$).

\section{The quasiasymptotic behavior of degree -1 and summability of Fourier}

series and integrals. In this section, we discuss the structure of the quasiasymptotic behavior of degree -1 within the context of summability of Fourier series and integrals. In particular, we obtain in an easy way the characterization of distributional point values [10] (i.e., the value of a distribution at a point in the sense of Łojasiewicz) of periodic functions given by R. Estrada in [6]. Some results of G. Walter [26, 27] are obtained by this method. We also discuss the case of jump behavior of distributions at a point [8, 21, 22].

We first specialize Theorem 2.7 at infinity for $k=1$ and the trivial slowly varying function. Recall the notion of limits of distributions in the Cesàro sense $[7,9]$. Let $f \in \mathcal{D}^{\prime}$, we write

$$
\lim _{x \rightarrow \infty} f(x)=\gamma \quad(\mathrm{C}, m),
$$

if there exists an $m$-primitive of $f$, say $F_{m}$, such that $F_{m}$ is a regular distribution in some 
interval of the form $[A, \infty)$, for some $A$, and satisfies the ordinary asymptotic relation

$$
F_{m}(x)=\gamma \frac{x^{m}}{m !}+o\left(x^{m}\right) \quad \text { as } x \rightarrow \infty .
$$

Note that if (9) holds for $F_{m}$, then it holds for all $m$-primitives of $f$. So, we may reformulate some parts of Theorem 2.7 as follows.

THEOREM 3.1. Let $f \in \mathcal{D}^{\prime}$, then

$$
f(\lambda x)=\gamma \delta(\lambda x)+\beta \text { p.v. }\left(\frac{1}{\lambda x}\right)+o\left(\frac{1}{\lambda}\right) \quad \text { as } \lambda \rightarrow \infty \text { in } \mathcal{D}^{\prime}
$$

if and only if there exists $m \in \mathbb{N}$ such that for a primitive $F$ of $f$, i.e., $F^{\prime}=f$, the following limit holds for each $a>0$

$$
\lim _{x \rightarrow \infty} F(a x)-F(-x)=\gamma+\beta \log a \quad(\mathrm{C}, m) .
$$

We now study the distributional jump behavior of a distribution at a point. Let $f \in \mathcal{D}^{\prime}$ and $x_{0} \in \mathbb{R}$, we say that $f$ has distributional jump behavior at $x=x_{0}$ if $f\left(x_{0}+x\right)$ has the following quasiasymptotic behavior at the origin

$$
f\left(x_{0}+\varepsilon x\right)=\gamma_{-} H(-x)+\gamma_{+} H(x) \quad \text { as } \varepsilon \rightarrow 0^{+} \text {in } \mathcal{D}^{\prime} .
$$

Note that if $\gamma_{-}=\gamma_{+}$we obtain the usual Eojasiewicz notion of the value of a distribution at point [10]. We may deal only with tempered distributions because we want to consider Fourier transform [17]. We will consider the following Fourier transform, $\hat{\phi}(x)=\int_{-\infty}^{\infty} \phi(t) e^{-i x t} \mathrm{~d} t$ for $\phi \in \mathcal{S}$, and as usual we define it on $\mathcal{S}^{\prime}$ by considering the transpose. If $f \in \mathcal{S}^{\prime}$ in $(10)$, then the asymptotic relation holds also in $\mathcal{S}^{\prime}[8,23,30]$. Hence if we apply Fourier transform to (10) and use the very well-known formulas for the Fourier transform of $H$, we obtain it is equivalent to

$$
e^{i \lambda x_{0} x} \hat{f}(\lambda x)=2 \pi d_{1} \delta(\lambda x)-i d_{2} \text { p.v. }\left(\frac{1}{\lambda x}\right)+o\left(\frac{1}{\lambda}\right) \quad \text { as } \lambda \rightarrow \infty \text { in } \mathcal{S}^{\prime},
$$

where $d_{1}=\left(\gamma_{+}+\gamma_{-}\right) / 2$ and $d_{2}=\gamma_{+}-\gamma_{-}$. Note that we can interpret $d_{2}=[f]_{x=x_{0}}$ as the jump of $f$ at $x=x_{0}$. In the following two subsections, for simplicity, we assume that $\hat{f}=\mu$ is a (regular) Borel measure, but the author remarks that these results hold, with a suitable interpretation, for general tempered distributions.

3.1. Characterization of the jump behavior by asymmetric Cesàro means. Let $f$ be as in the previous discussion, that is, $f \in \mathcal{S}^{\prime}, f$ has the distributional jump behavior (10), and the Fourier transform of $f$ is a Borel measure $\hat{f}=\mu$. We can apply Theorem 3.1 to 11 and deduce that $f \in \mathcal{S}^{\prime}$ has the jump behavior 10 if and only if there exists $m \in \mathbb{N}$ such that for each $a>0$

$$
\lim _{x \rightarrow \infty} \frac{1}{2 \pi} \int_{-x}^{a x} e^{i x_{0} t} \mathrm{~d} \mu(t)=d_{1}+\frac{d_{2}}{2 \pi i} \log a \quad(\mathrm{C}, m) .
$$

If we introduce the means functions

$$
\phi_{a}^{m}(t)=(1+t)^{m} \chi_{[-1,0]}+(1-t / a)^{m} \chi_{[0, a]}(t),
$$

a direct calculation shows that 12 is equivalent to

$$
\lim _{x \rightarrow \infty} \frac{1}{2 \pi} \int_{-\infty}^{\infty} e^{i x_{0} t} \phi_{a}^{m}\left(\frac{t}{x}\right) \mathrm{d} \mu(t)=d_{1}+\frac{d_{2}}{2 \pi i} \log a
$$


furthermore, the last relation holds uniformly for $a$ on compact subsets of $(0, \infty)$. This characterizes completely the jump behavior in terms of Cesàro summability of asymmetric trigonometric integrals when the Fourier transform is a Borel measure.

REMARK 3.2. More general results, valid even if $\hat{f}$ is not a Borel measure, have been given in $[21,22]$. In the case of Łojasiewicz point values, a pointwise inversion formula is given by using the concept of special values of distributional evaluations in the Cesàro sense, the details can be found in the cited references.

3.2. Abel means. We now discuss the case of Abel summability. Let $f$ be again such that $f \in \mathcal{S}^{\prime}, f$ has the distributional jump behavior 10 , and the Fourier transform $\hat{f}=\mu$ is a Borel measure. Let $\mu=\mu_{-}+\mu_{+}$be any decomposition of $\mu$ as the sum of two Borel measures having supports in $(-\infty, 0]$ and $[0, \infty)$, respectively. Then

$$
U(z)=\frac{1}{2 \pi} \int_{-\infty}^{0} e^{i \bar{z} t} \mathrm{~d} \mu_{-}(t)+\frac{1}{2 \pi} \int_{0}^{\infty} e^{i z t} \mathrm{~d} \mu_{+}(t), \quad \Im m z>0,
$$

is a harmonic representation of $f$ in the upper half-plane, that is,

$$
\lim _{y \rightarrow 0^{+}} U(x, y)=f(x),
$$

where the last limit is taken in $\mathcal{D}^{\prime}[3]$. We shall study the behavior of $U$ when approaching $x_{0}$ through any line in the upper half-plane $\Im m z>0$. Note that because of the results of [8], any harmonic representation will have the same behavior at the boundary point. We remark this problem was studied in [8] by using the Poisson kernel. Because of (8) in Theorem 2.7, we can choose the measures in the decomposition having the following expansions,

$$
e^{i \lambda x_{0} x} \mu_{+}(\lambda x)=\left(b(\lambda)+\pi d_{1}\right) \delta(\lambda x)+\frac{d_{2}}{i \lambda} \operatorname{Pf}\left(\frac{H(x)}{x}\right)+o\left(\frac{1}{\lambda}\right)
$$

and

$$
e^{i \lambda x_{0} x} \mu_{-}(\lambda x)=\left(-b(\lambda)+\pi d_{1}\right) \delta(\lambda x)+\frac{d_{2}}{i \lambda} \operatorname{Pf}\left(\frac{H(-x)}{x}\right)+o\left(\frac{1}{\lambda}\right),
$$

as $\lambda \rightarrow \infty$ in $\mathcal{S}^{\prime}$. So, we have that if the angle $\theta$ stays in a compact subset of $(-\pi / 2, \pi / 2)$, the following limit holds uniformly in $\theta$,

$$
\begin{aligned}
U\left(x_{0}+\frac{\tan \theta}{\lambda}, \frac{1}{\lambda}\right) & =\left\langle\lambda e^{i \lambda x_{0} x} \mu_{-}(\lambda x), \frac{e^{(1+i \tan \theta) x}}{2 \pi}\right\rangle+\left\langle\lambda e^{i \lambda x_{0} x} \mu_{+}(\lambda x), \frac{e^{(-1+i \tan \theta) x}}{2 \pi}\right\rangle \\
& =d_{1}+\frac{d_{2}}{\pi} \int_{0}^{\infty} \sin (x \tan \theta) \frac{e^{-x}}{x} \mathrm{~d} x+o(1), \quad \text { as } \lambda \rightarrow \infty .
\end{aligned}
$$

Using Parseval's relation in the last integral and making the simplifications one has that

$$
\int_{0}^{\infty} \sin (x \tan \theta) \frac{e^{-x}}{x} \mathrm{~d} x=\frac{1}{2 i} \int_{-\tan \theta}^{\tan \theta} \frac{\mathrm{d} t}{t-i}=\theta .
$$

Therefore, we have obtained

$$
\lim _{\lambda \rightarrow \infty} U\left(x_{0}+\frac{\tan \theta}{\lambda}, \frac{1}{\lambda}\right)=d_{1}+\frac{\theta}{\pi} d_{2} .
$$


If we take $\theta=0$ in $(13)$, we obtain a pointwise Fourier inversion formula in terms of Abel means of the trigonometric integral

$$
\lim _{\varepsilon \rightarrow 0^{+}} \frac{1}{2 \pi} \int_{-\infty}^{\infty} e^{-\varepsilon|x|} e^{i x_{0} x} d \mu(x)=\frac{\gamma_{-}+\gamma_{+}}{2} .
$$

Note that it is not necessary to assume that $\hat{f}$ is a Borel measure, in such a case we decompose $\hat{f}=\hat{f}_{-}+\hat{f}_{+}$, where they satisfy the properties of $\mu_{ \pm}$, and use the harmonic representation

so we obtain that

$$
U(z)=\frac{1}{2 \pi}\left\langle\hat{f}_{-}(t), e^{i \bar{z} t}\right\rangle+\frac{1}{2 \pi}\left\langle\hat{f}_{+}(t), e^{i z t}\right\rangle
$$

$$
\lim _{z \rightarrow x_{0}, z \in l_{\theta}} U(z)=d_{1}+\frac{\theta}{\pi} d_{2}
$$

where $l_{\theta}$ denotes the ray in the upper half-plane starting from $x_{0}$ and making an angle $\theta$ with the line $x=x_{0}$, here $-\pi / 2<\theta<\pi / 2$. This relation holds uniformly for $\theta$ in compact subsets of $(-\pi / 2, \pi / 2)$.

3.3. Case of Fourier series of distributions. We may specialize the results of the last two subsections to $2 \pi$-periodic distributions. Let $f(x)=\sum_{-\infty}^{\infty} c_{n} e^{i n x}$, where the series is assumed to converge in $\mathcal{S}^{\prime}$. Since

$$
\hat{f}(x)=2 \pi \sum_{-\infty}^{\infty} c_{n} \delta(x-n)
$$

we immediately obtain that $f$ satisfies 10 if and only if there is an $m \in \mathbb{N}$ such that for each $a>0$

$$
\lim _{x \rightarrow \infty} \sum_{-\infty}^{\infty} c_{n} e^{i x_{0} n} \phi_{a}^{m}\left(\frac{n}{x}\right)=d_{1}+\frac{d_{2}}{2 \pi i} \log a .
$$

Let $l_{\theta}$ denote the ray in the upper half-plane starting from $x_{0}$ and making an angle $\theta$ with the line $x=x_{0}$, here $-\pi / 2<\theta<\pi / 2$. Then from $(13)$, one has that

$$
\lim _{\xi \rightarrow x_{0}, \xi \in l_{\theta}} \sum_{-\infty}^{-1} c_{n} e^{i n \bar{\xi}}+\sum_{0}^{\infty} c_{n} e^{i n \xi}=d_{1}+\frac{\theta}{\pi} d_{2} .
$$

If we write the cosines and sines series, $f(x)=\sum_{n=0}^{\infty} a_{n} \cos (n x)+b_{n} \sin (n x)$, then 15 takes the form

$$
\lim _{\xi \rightarrow x_{0}, \xi \in l_{\theta}} \sum_{n=0}^{\infty} a_{n} \cos (n \xi)+b_{n} \sin (n \xi)=d_{1}+\frac{\theta}{\pi} d_{2},
$$

both (15) and (16) hold uniformly for $\theta$ in compact subsets of $(-\pi / 2, \pi / 2)$.

REMARK 3.3. The characterization (14) appears in [21, 22]. It generalizes R. Estrada's characterization from [6]; indeed, when $f$ has a distributional point value at $x_{0}$, say $f\left(x_{0}\right)=\gamma$ in $\mathcal{D}^{\prime}$, then $d_{1}=\gamma$ and $d_{2}=0$ and the logarithmic term disappears, this is precisely the cited result from [6]. If one takes $\theta=0$ in $[16]$, one obtains

$$
\lim _{r \rightarrow 1^{-}} \sum_{n=0}^{\infty}\left(a_{n} \cos n x_{0}+b_{n} \sin n x_{0}\right) r^{n}=d_{1},
$$


which in the case of Łojasiewicz point values is the result obtained for the Abel-Poisson means of the series in [27] by G. Walter.

4. Some properties of the quasiasymptotics at infinity. Sometimes is very useful to have the right of evaluating (2) in more test functions than in $\mathcal{S}$. This section is dedicated to give some conditions over the test functions which guarantee one can do this. We need the following definition.

Definition 4.1. Let $\phi \in \mathcal{E}$ and $\beta \in \mathbb{R}$. We say that

$$
\phi(x)=O\left(|x|^{\beta}\right) \quad \text { strongly as }|x| \rightarrow \infty,
$$

if for each $m \in\{0,1,2, \ldots\}$

$$
\phi^{(m)}(x)=O\left(|x|^{\beta-m}\right) \quad \text { as }|x| \rightarrow \infty .
$$

The set of $\phi$ 's satisfying Definition 4.1 for a particular $\beta$ forms the space $\mathcal{K}_{\beta}$ which we topologize in the obvious way [9]. These spaces and their dual spaces are very important in the theory of asymptotic expansions of distributions [9]. In fact, if we set $\mathcal{K}=\bigcup \mathcal{K}_{\beta}$ (the union having a topological meaning), we obtain that $\mathcal{K}^{\prime}$ is the space of distributional small distributions at infinity [7, 9], they satisfy the moment asymptotic expansion at infinity [9]. We need the following lemma whose proof can be found in [20, Proposition 2.11].

LEMMA 4.2. If $b$ is associate asymptotically homogeneous of degree 0 at infinity with respect to the slowly varying function $L$, then $b(x)=O\left(x^{\sigma}\right)$ as $x \rightarrow \infty$, for any $\sigma>0$.

The next theorem shows that if $f$ has quasiasymptotics at infinity, then the distributional evaluation of $f$ at $\phi \in \mathcal{K}_{\beta}$ makes sense under some conditions on $\beta$, specifically, we show that $f$ has extensions to some of the spaces $\mathcal{K}_{\beta}$.

THEOREM 4.3. Let $f \in \mathcal{D}^{\prime}$ have quasiasymptotic behavior of degree $\alpha$ at infinity with respect to the slowly varying function $L$. If $\alpha+\beta<-1$, then $f$ admits an extension to $\mathcal{K}_{\beta}$.

Proof. Let $\sigma>0$ such that $\alpha+\beta+\sigma<-1$, then from Theorem 2.5, Theorem 2.7 and Lemma 4.2 we deduce that there exist $m \in \mathbb{N}$ and a continuous $m$-primitive of $f$, say $F$, such that

$$
F(x)=O\left(|x|^{m+\alpha+\sigma}\right) \quad \text { as }|x| \rightarrow \infty .
$$

Notice that here we have used that $L(x)=O\left(x^{\sigma}\right)$ as $x \rightarrow \infty$ [18]. So it is evident that an extension of $f$ to $\mathcal{K}_{\beta}$ is given by

$$
\langle f(x), \phi(x)\rangle=(-1)^{m} \int_{-\infty}^{\infty} F(x) \phi^{(m)}(x) \mathrm{d} x, \quad \phi \in \mathcal{K}_{\beta},
$$

which in view of $(17)$ and $\sqrt{18}$ is well-defined and defines an element of $\mathcal{K}_{\beta}^{\prime}$.

We now show that the quasiasymptotic behavior remains valid in $\mathcal{K}_{\beta}^{\prime}$, with the assumption under $\beta$ imposed in Theorem 4.3. 
THEOREM 4.4. Let $f \in \mathcal{D}^{\prime}$ have quasiasymptotic behavior at $\infty$ of degree $\alpha$ with respect to a slowly varying function $L$. If $\alpha+\beta<-1$, then $f$ has an extension to $\mathcal{K}_{\beta}$ which has the same quasiasymptotics in $\mathcal{K}_{\beta}^{\prime}$.

Proof. The proof is similar to that of [23, Theorem 6.1] with some modifications in the estimates. We will use the extension of $f$ found in Theorem 4.3. We shall divide the proof into two cases: $\alpha \notin\{-1,-2,-3, \ldots\}$ and $\alpha \in\{-1,-2,-3, \ldots\}$.

Suppose its degree is $\alpha \notin\{-1,-2,-3, \ldots\}$ and

$$
f(\lambda x)=C_{-} L(\lambda) \frac{(\lambda x)_{-}^{\alpha}}{\Gamma(\alpha+1)}+C_{+} L(\lambda) \frac{(\lambda x)_{+}^{\alpha}}{\Gamma(\alpha+1)}+o\left(\lambda^{\alpha} L(\lambda)\right) \quad \text { as } \lambda \rightarrow \infty,
$$

in $\mathcal{D}^{\prime}$. Find $\sigma>0$ such that $\alpha+\beta+\sigma<-1$. Then, there are an $m$ such that $m+\alpha>0$ and a continuous $m$-primitive $F$ of $f$ such that

$$
F(x)=\frac{x^{m}|x|^{\alpha}}{\Gamma(m+\alpha+1)} L(|x|)\left(C_{-} H(-x)+C_{+} H(x)\right)+o\left(|x|^{m+\alpha} L(|x|)\right),
$$

$x \rightarrow \infty$. We recall that $H$ denotes the Heaviside function. We make the usual assumptions over $L$, assume that $L$ is positive, defined and continuous in $(0, \infty)$ and there exists $M_{1}>0$ such that

$$
\frac{L(\lambda x)}{L(\lambda)} \leq M_{1} \max \left\{x^{\sigma}, x^{-\sigma}\right\}, \quad \lambda \geq 1, \quad x \in(0, \infty),
$$

in [23, Section 2] are the reason why these assumptions can be always made (see Potter's Theorem [2, p. 25] also). Let $\phi \in \mathcal{K}_{\beta}$. Then we can decompose $\phi=\phi_{1}+\phi_{2}+\phi_{3}$, where $\operatorname{supp} \phi_{1} \subseteq(-\infty, 1]$, supp $\phi_{2}$ is compact and supp $\phi_{3} \subseteq[1, \infty)$. Observe that since $\phi_{2} \in \mathcal{D}$ we have that

$$
\left\langle f(\lambda x), \phi_{2}(x)\right\rangle \sim C_{-} \lambda^{\alpha} L(\lambda)\left\langle\frac{x_{-}^{\alpha}}{\Gamma(\alpha+1)}, \phi_{2}(x)\right\rangle+C_{+} \lambda^{\alpha} L(\lambda)\left\langle\frac{x_{+}^{\alpha}}{\Gamma(\alpha+1)}, \phi_{2}(x)\right\rangle,
$$

as $\lambda \rightarrow \infty$. If we want to show 20 for $\phi$, it is enough to show it for $\phi_{3}$ placed instead of $\phi_{2}$ in the relation because by symmetry it would follow for $\phi_{1}$ and hence for $\phi$. Set

$$
G(x)=\frac{F(x)}{x^{\alpha+m} L(x)} \quad \text { for } x \geq 1,
$$

then

$$
\lim _{x \rightarrow \infty} G(x)=\frac{C_{+}}{\Gamma(\alpha+m+1)} .
$$

So, we can find a constant $M_{2}>0$ such that

$$
|G(x)|<M_{2}, \text { globally. }
$$

Relation (21) together with 19 show that for $\lambda \geq 1$,

$$
\left|G(\lambda x) \frac{L(\lambda x)}{L(\lambda)} x^{\alpha+m} \phi_{3}^{(m)}(x)\right| \leq M_{1} M_{2} x^{\alpha+m+\sigma}\left|\phi_{3}^{(m)}(x)\right| H(x-1) .
$$

Since $\phi_{3} \in \mathcal{K}_{\beta}$, the right hand side of the last estimate belongs to $L^{1}(\mathbb{R})$ and thus we can 
use the Lebesgue dominated convergence theorem to obtain

$$
\begin{aligned}
\lim _{\lambda \rightarrow \infty} \frac{1}{\lambda^{\alpha} L(\lambda)}\left\langle f(\lambda x), \phi_{3}(x)\right\rangle & =\lim _{\lambda \rightarrow \infty}(-1)^{m} \int_{0}^{\infty} G(\lambda x) \frac{L(\lambda x)}{L(\lambda)} x^{\alpha+m} \phi_{3}^{(m)}(x) \mathrm{d} x \\
& =(-1)^{m} \frac{C_{+}}{\Gamma(\alpha+m+1)} \int_{0}^{\infty} x^{\alpha+m} \phi_{3}^{(m)}(x) \mathrm{d} x \\
& =C_{+}\left\langle\frac{x_{+}^{\alpha}}{\Gamma(\alpha+1)}, \phi_{3}(x)\right\rangle .
\end{aligned}
$$

This shows the result in the case $\alpha \notin\{-1,-2,-3, \ldots\}$.

We now aboard the case $\alpha=-k, k \in \mathbb{N}$. Assume that

$$
f(\lambda x)=\gamma \lambda^{-k} L(\lambda) \delta^{(k-1)}(x)+\beta \lambda^{-k} L(\lambda) x^{-k}+o\left(\lambda^{-k} L(\lambda)\right)
$$

as $\lambda \rightarrow \infty$ in $\mathcal{D}^{\prime}$. As in the last case, it suffices to assume that $\phi \in \mathcal{K}_{\beta}, \operatorname{supp} \phi \subseteq[1, \infty)$ and show that

$$
\lim _{\lambda \rightarrow \infty} \frac{\lambda^{k}}{L(\lambda)}\langle f(\lambda x), \phi(x)\rangle=\beta \int_{1}^{\infty} \frac{\phi(x)}{x^{k}} \mathrm{~d} x .
$$

We may proceed as in the previous case to apply the structural theorem, but we rather reduce it to the previous situation. So, set $g(x)=x^{k} f(x)$. Then

$$
g(\lambda x)=\beta L(\lambda)+o(L(\lambda)) \quad \text { as } \lambda \rightarrow \infty \text { in } \mathcal{D}^{\prime} .
$$

But $\phi \in \mathcal{K}_{\beta}$ implies $\phi(x) / x^{k} \in \mathcal{K}_{\beta-k}$ then since the degree of the quasiasymptotic behavior of $g$ is 0 , last case implies that 222 is valid in $\mathcal{K}_{\beta-k}^{\prime}$ because $\beta-k<-1$, therefore

$$
\lim _{\lambda \rightarrow \infty} \frac{\lambda^{k}}{L(\lambda)}\langle f(\lambda x), \phi(x)\rangle=\lim _{\lambda \rightarrow \infty} \frac{1}{L(\lambda)}\left\langle g(\lambda x), \frac{\phi(x)}{x^{k}}\right\rangle=\beta \int_{1}^{\infty} \frac{\phi(x)}{x^{k}} \mathrm{~d} x .
$$

This completes the proof of Theorem 4.4.

5. Structure of quasiasymptotically bounded distributions. This section is intended to study the structure of the distributional relation

$$
f(\lambda x)=O(\rho(\lambda)),
$$

where here $\lambda \rightarrow \infty$ or $\lambda \rightarrow 0^{+}$and $\rho$ is a regularly varying function. Distributions satisfying this relation will be called quasiasymptotically bounded distributions, we make this more precise in the following definition.

DEFINITION 5.1. Let $L$ be a slowly varying function at infinity (respectively at the origin). We say that $f \in \mathcal{D}^{\prime}$ is quasiasymptotically bounded at infinity (at the origin) in $\mathcal{D}^{\prime}$ with respect to $\lambda^{\alpha} L(\lambda), \alpha \in \mathbb{R}$, if

$$
\langle f(\lambda x), \phi(x)\rangle=O\left(\lambda^{\alpha} L(\lambda)\right) \quad \text { as } \lambda \rightarrow \infty \forall \phi \in \mathcal{D},
$$

(respectively $\lambda \rightarrow 0^{+}$). If (23) holds, it is also said that $f$ is quasiasymptotically bounded of degree $\alpha$ at infinity (at the origin) with respect to the slowly varying function $L$. We express (23) by

$$
f(\lambda x)=O\left(\lambda^{\alpha} L(\lambda)\right) \quad \text { as } \lambda \rightarrow \infty \text { in } \mathcal{D}^{\prime},
$$

(respectively $\lambda \rightarrow 0^{+}$). 
In the case at infinity, since $L(\lambda)=O\left(\lambda^{\sigma}\right)$ as $\lambda \rightarrow \infty$, for any $\sigma>0$, it follows immediately from $[9$, Theorem 6.6.1] that $f$ must be a tempered distribution. Note that in analogy to the quasiasymptotic behavior of distributions, we may talk about (24) in other spaces of distributions. It will follow from our structural theorem that actually the relation holds in $\mathcal{S}^{\prime}$. The case at the origin is related to the problem of extension of distributions from $\mathbb{R} \backslash\{0\}$ to $\mathbb{R}$. Indeed, if $f \in \mathcal{D}^{\prime}(\mathbb{R} \backslash\{0\})$ and $(23)$ holds for all $\phi \in \mathcal{D}(\mathbb{R} \backslash\{0\})$, it follows from [7, Theorem 6.9.1] that $f$ admits an extension to $\mathbb{R}$.

We now proceed to obtain the structure of quasiasymptotically bounded distributions. For this aim, the program established in [20, 23] will be followed. We will integrate the relation (24) and the coefficients of this integration will satisfy the properties of the following definition.

Definition 5.2. A function $b$ is said to be asymptotically homogeneously bounded of degree $\alpha$ at infinity with respect to the slowly varying function $L$ if it is measurable and defined in some interval $[A, \infty), A>0$, and for each $a>0$

$$
b(a x)=a^{\alpha} b(x)+O(L(x)), \quad x \rightarrow \infty .
$$

Similarly, one defines asymptotically homogeneously bounded functions at the origin. Our first goal is to study the asymptotic properties of this class of functions. From the results of [18, Section 2.4], one has that 25 must hold uniformly for $a$ in compact subsets of $(0, \infty)$. Most of the proofs of the following results are the analog to those for asymptotically homogeneous functions by replacing the $o$ symbol by the $O$ symbol and making obvious modifications to the estimates. Therefore, they will be omitted; we refer to $[20,23]$ and leave to the reader the details of such modifications.

Proposition 5.3. Let $b$ be asymptotically homogeneously bounded at infinity (at the origin) with respect to the slowly varying function $L$. If the degree is negative (respectively positive), then $b(x)=O(L(x))$, as $x \rightarrow \infty\left(x \rightarrow 0^{+}\right)$.

Proposition 5.4. Let $b$ be asymptotically homogeneously bounded at infinity (at the origin) with respect to the slowly varying function $L$. If the degree is positive (respectively negative), then there exits a constant $\gamma$ such that $b(x)=\gamma x^{\alpha}+O(L(x))$, as $x \rightarrow \infty$ $\left(x \rightarrow 0^{+}\right)$.

Note that for the case at infinity since $L(x)=O\left(x^{\sigma}\right)$ as $x \rightarrow \infty$, for any $\sigma>0$, then any asymptotically homogeneously bounded function of degree 0 at infinity satisfies that $b(x) / x^{\sigma}$ is asymptotically homogeneously bounded of degree $-\sigma$ with respect to the trivial slowly varying function $L \equiv 1$ and hence by Proposition 5.3 it satisfies $b(x)=O\left(x^{\sigma}\right)$ as $x \rightarrow \infty$. Then, for large argument, it is a regular tempered distribution. Similarly, any asymptotically homogeneously bounded function of degree 0 at the origin satisfies $b(x)=O\left(x^{-\sigma}\right)$ as $x \rightarrow 0^{+}$, for any $\sigma>0$, consequently it is a distribution for small argument. The proof of the next proposition is totally analogous to those of [20, Theorem 2.12] and [23, Theorem 3.8] therefore will be omitted again.

Proposition 5.5. Let $b$ be asymptotically homogeneously bounded of degree zero at infinity (at the origin) with respect to the slowly varying function $L$. Suppose that $b$ is locally integrable on $[A, \infty)$ (respectively $(0, A])$. We denote by $b(x) H(x-A)$ the regular 
distribution defined by

$$
\langle b(x) H(x-A), \phi(x)\rangle=\int_{A}^{\infty} b(x) \phi(x) \mathrm{d} x,
$$

(respectively the distribution $b(x)(H(x)-H(x-A)))$. Then

$$
b(\lambda x) H(\lambda x-A)=b(\lambda) H(x)+O(L(\lambda)) \quad \text { as } \lambda \rightarrow \infty \text { in } \mathcal{S}^{\prime},
$$

(respectively $b(\lambda x)(H(x)-H(\lambda x-A))$ as $\lambda \rightarrow 0^{+}$in $\left.\mathcal{D}^{\prime}\right)$.

Corollary 5.6. Let $b$ be an asymptotically homogeneously bounded function of degree 0 at infinity (at the origin) with respect to $L$. Then, there exists $c \in \mathcal{C}^{\infty}(0, \infty)$, being asymptotically homogeneously bounded of degree 0, such that $b(x)=c(x)+O(L(x))$ as $x \rightarrow \infty$ (respectively as $x \rightarrow 0^{+}$). Moreover, we may choose $c$ vanishing in a neighborhood of $0(\infty)$.

Proof. We only show the assertion at infinity, the case at the origin is similar. Find $B$ such that $b$ is locally bounded in $[B, \infty)$. Take $\phi \in \mathcal{D}^{\prime}$ supported in $(0, \infty)$ such that $\int_{0}^{\infty} \phi(t) \mathrm{d} t=1$ and set $c(x)=\int_{B / x}^{\infty} b(x t) \phi(t) \mathrm{d} t$. The corollary now follows from Proposition 5.5.

The main connection between quasiasymptotically bounded distributions and asymptotically homogeneously bounded functions is given in the next proposition, again the proof will be omitted since it is analogous to those of [20, Proposition 2.5] and [23, Proposition 3.1].

Proposition 5.7. Let $f \in \mathcal{D}^{\prime}$ be quasiasymptotically bounded of degree $\alpha$ at infinity (at the origin) with respect to the slowly varying function $L$. Let $m \in \mathbb{N}$. Then, for any given $F_{m}$, an $m$-primitive of $f$ in $\mathcal{D}^{\prime}$, there exist functions $b_{0}, \ldots, b_{m-1}$, continuous on $(0, \infty)$, such that

$$
F_{m}(\lambda x)=\sum_{j=0}^{m-1} \lambda^{\alpha+m} b_{j}(\lambda) \frac{x^{m-1-j}}{(m-1-j) !}+O\left(\lambda^{\alpha+m} L(\lambda)\right) \quad \text { in } \mathcal{D}^{\prime},
$$

as $\lambda \rightarrow \infty$ (respectively $\lambda \rightarrow 0^{+}$), where each $b_{j}$ is asymptotically homogeneously bounded of degree $-\alpha-j-1$ with respect to $L$.

Thus we obtain from Proposition 5.3, Proposition 5.4 and Proposition 5.7 our first structural theorem.

TheOrem 5.8. Let $f \in \mathcal{D}^{\prime}$ and $\alpha \notin \mathbb{Z}_{-}$. Then $f$ is quasiasymptotically bounded of degree $\alpha$ at infinity (at the origin) with respect to the slowly varying function $L$ if and only if there exist $m \in \mathbb{N}, m+\alpha>0$, and a continuous (continuous near 0 ) m-primitive $F$ of $f$ such that

$$
F(x)=O\left(|x|^{m+\alpha} L(|x|)\right),
$$

as $|x| \rightarrow \infty$ (respectively $x \rightarrow 0)$ in the ordinary sense. Moreover, in the case at infinity, $f$ is quasiasymptotically bounded of degree $\alpha$ with respect to $L$ in $\mathcal{S}^{\prime}$.

Proof. We only discuss the case at infinity, the proof of the assertion at the origin is similar to this case. It follows from Proposition 5.7, Proposition 5.3 and Proposition 5.4 
that given $m \in \mathbb{N}$ and an $m$-primitive $F_{m}$, there is a polynomial $p_{m-1}$ of degree at most $m-1$ such that

$$
F_{m}(\lambda x)=p_{m-1}(\lambda x)+O\left(\lambda^{\alpha+m} L(\lambda)\right) \quad \text { as } \lambda \rightarrow \infty \text { in } \mathcal{D}^{\prime} .
$$

From the definition of boundedness in $\mathcal{D}^{\prime}$, it follows that there is an $m>-\alpha$ such that 27) holds uniformly for $x \in[-1,1]$. We let $F=F_{m}-p_{m-1}$, so by taking $x=-1, x=1$ and replacing $\lambda$ by $x$ in (27) we obtain (26). The converse follows by observing that 26. implies that $F(\lambda x)=O\left(\lambda^{\alpha+m} L(\lambda)\right)$ in $\mathcal{S}^{\prime}$ which gives the result after differentiating $m$-times.

We now analyze the other cases.

THEOREM 5.9. Let $f \in \mathcal{D}^{\prime}$ and let $k$ be a positive integer. Then $f$ is quasiasymptotically bounded of degree $-k$ at infinity (at the origin) with respect to $L$ if and only if there exist $m>k \in \mathbb{N}$, an asymptotically homogeneously bounded function $b$ of degree 0 at infinity (at the origin) with respect to $L$, and a continuous (continuous near 0) m-primitive $F$ of $f$ such that

$$
F(x)=b(|x|) x^{m-k}+O\left(|x|^{m-k} L(|x|)\right),
$$

as $|x| \rightarrow \infty(x \rightarrow 0)$. Moreover (28) is equivalent to have

$$
a^{k-m} F(a x)-(-1)^{m-k} F(-x)=O\left(x^{m-k} L(x)\right),
$$

as $x \rightarrow \infty\left(x \rightarrow 0^{+}\right)$, for each $a>0$. In the case at infinity, it follows that $f$ is quasiasymptotically bounded of degree $-k$ with respect to $L$ in $\mathcal{S}^{\prime}$.

Proof. Again we only give the proof of the assertion at infinity, the case at the origin is similar. If $f(\lambda x)=O\left(\lambda^{-k} L(\lambda)\right)$ in $\mathcal{D}^{\prime}$, then after $k-1$ integrations Proposition 5.7 and Proposition 5.4 provide us of a $(k-1)$-primitive of $f$ which is quasiasymptotically bounded of degree -1 at infinity with respect to $L$, hence we may assume that $k=1$. Next, Proposition 5.7, Proposition 5.3 and the definition of boundedness in $\mathcal{D}^{\prime}$ give to us the existence of an $m>1$, an asymptotically homogeneously bounded function of degree -1 with respect to $L$ and an $m$-primitive $F$ of $f$ such that $F(\lambda x)$ is continuous for $x \in[-1,1]$ (hence $F$ is continuous on $\mathbb{R}$ ) and $F(\lambda x)=\lambda^{m-1} b(\lambda) x^{m-1}+O\left(\lambda^{m-1} L(\lambda)\right)$ as $\lambda \rightarrow \infty$ uniformly for $x \in[-1,1]$, by taking $x=-1, x=1$ and replacing $\lambda$ by $x$ one gets (28). Assume (28), by using Corollary 5.6, we may assume that $b$ is locally integrable on $[0, \infty)$, this allows the application of Proposition 5.5 to deduce that $F(\lambda x)=\lambda^{m-1} b(\lambda) x^{m-1}+$ $O\left(\lambda^{m-1} L(\lambda)\right)$ as $\lambda \rightarrow \infty$ in $\mathcal{S}^{\prime}$ and hence the converse follows by differentiating $m$-times. That (28) implies 29 is a simple calculation; conversely, setting $b(x)=x^{k-m} F(x)$ for $x>0$, one obtains $(28)$.

This section ends with three remarks concerning some consequences of the structural theorems for quasiasymptotically bounded distributions.

REMARK 5.10. Even if not initially assumed, the proof of Theorem 5.9 forces $(29)$ to hold uniformly for $a$ on compact subsets of $(0, \infty)$.

REMARK 5.11. The results of Section 4 are also valid for quasiasymptotically bounded distributions. Indeed, let $f \in \mathcal{D}^{\prime}$ be quasiasymptotically bounded of degree $\alpha$ at infinity with respect to the slowly varying function $L$. If $\alpha+\beta<-1$, then $f$ has an extension to 
$\mathcal{K}_{\beta}$, the proof of this assertion is identical to that of Theorem 4.3. Moreover, if one follows the proof of Theorem 4.4, one finds that it is even easier to show that such an extension of $f$ is quasiasymptotically bounded at infinity with respect to $L$, with the same degree, in $\mathcal{K}_{\beta}^{\prime}$ provided that $\alpha+\beta<-1$.

REMARK 5.12. This remark is the analog to Remark 2.6 in the case of quasiasymptotically bounded distributions. Let $\alpha \notin \mathbb{Z}_{-}$. If we only assume that $f \in \mathcal{D}^{\prime}(\mathbb{R} \backslash\{0\})$ is quasiasymptotically bounded of degree $\alpha$ at the origin with respect to $L$ in $\mathcal{D}^{\prime}(\mathbb{R} \backslash\{0\})$, then the arguments given in this section lead to the existence of an $m>-\alpha$ and a distribution $F$, which is a continuous function near 0 , such that $F(x)=O\left(|x|^{m+\alpha} L(|x|)\right)$ and $F^{(m)}=f$ on $\mathcal{D}^{\prime}(\mathbb{R} \backslash\{0\})$. Hence $F^{(m)}$ is an extension of $f$ to $\mathbb{R}$ which is quasiasymptotically bounded in $\mathcal{D}^{\prime}$; the next argument shows that if $\alpha>-1$, then the quasiasymptotically bounded extension of degree $\alpha$ is unique. If $f$ is already defined on $\mathbb{R}$ but only satisfies $f(\lambda x)=O\left(\lambda^{\alpha} L(\lambda)\right)$ as $\lambda \rightarrow 0^{+}$in $\mathcal{D}^{\prime}(\mathbb{R} \backslash\{0\})$, then there are constants $a_{0}, \ldots, a_{n}$ such that $f+\sum_{j=0}^{n} a_{j} \delta^{(j)}$ is quasiasymptotically bounded at the origin with respect to $\lambda^{\alpha} L(\lambda)$, these constants are unique if $\alpha>-1$. These properties are not satisfied for $\alpha \in \mathbb{Z}_{-}$as shown by the example $f(x)=g^{\prime}(x)$, where $g(x)=\log ^{2} x H(x)$. Indeed, $g(x)$ is asymptotically homogeneously bounded of degree 0 with respect to $\log x^{-1}$, hence $g(\lambda x)=g(\lambda) H(x)+O\left(\log \lambda^{-1}\right)$ as $\lambda \rightarrow 0^{+}$in $\mathcal{D}^{\prime}$, differentiating the last expression one has that $f(\lambda x)=\log ^{2} \lambda \delta(\lambda x)+O\left(\lambda^{-1} \log \lambda^{-1}\right)$ as $\lambda \rightarrow 0^{+}$in $\mathcal{D}^{\prime}$, therefore $f(\lambda x)=O\left(\lambda^{-1} \log \lambda^{-1}\right)$ as $\lambda \rightarrow 0^{+}$in $\mathcal{D}^{\prime}(\mathbb{R} \backslash\{0\})$ but it is impossible to find constants $a_{0}, \ldots, a_{n}$ such that $f+\sum_{j=0}^{n} a_{j} \delta^{(j)}$ be quasiasymptotically bounded at the origin with respect to $\lambda^{-1} \log \lambda^{-1}$. A counterexample for $\alpha=-k$ is constructed by considering $g^{(k)}$.

6. Open problems. In this last section, we pose two open problems concerning the quasiasymptotics of Schwartz distributions. The first problem is one-dimensional while the second one is multidimensional.

6.1. First open problem. It is known that for quasiasymptotic behaviors the optimal class to work with is the class of regularly varying functions. However, for quasiasymptotic boundedness one can still define the relations $f(\lambda x)=O(\rho(\lambda))$ and $f(\lambda x)=o(\rho(\lambda))$ even if $\rho$ is not a regularly varying function; furthermore, one may take any eventually positive function $\rho$. It is clear that if we do not impose restrictions over the function $\rho$, not too much can be said about the structure of these distributional relations. Since experience has shown that the structure of these type of asymptotic relations plays a very important role in the application of the notion, one may restrict the definition of quasiasymptotic boundedness to $\rho$ in classes of functions that allow one to describe the structure. Actually, this has been done in this paper for $\rho$ in the class of regularly varying functions. It seems that classes such as extended regularly varying functions or O-regularly varying functions [2] could be adequate for this purpose.

OPEn PROBLEM: Find the optimal classes of functions to work with quasiasymptotic boundedness.

6.2. Second open problem. The structure of quasiasymptotics in the multidimensional case is still an open question. Most known results are only valid under restrictions 
over the support of the distribution; some samples of these results can be found in [16, 24]. The recent work of Drozhzhinov and Zavialov [5] is of relevance for this open question. Their results suggest that spherical representations may be a path to follow in order to find an answer to such an important question.

Open PROBLEM: Find the complete structure of quasiasymptotic behaviors of distributions in the multidimensional case.

Acknowledgments. The author gratefully acknowledges support by the Louisiana State Board of Regents grant LEQSF(2005-2007)-ENH-TR-21. He also thanks the organizing committee of the International Conference on Generalized Functions GF07 for providing him a partial financial support to participate in the conference and present the preliminary version of this paper.

\section{References}

[1] J. M. Ash, P. Erdős and L. A. Rubel, Very slowly varying functions, Aequationes Math. 10 (1974), 1-9.

[2] N. H. Bingham, C. M. Goldie and J. L. Teugels, Regular Variation, Encyclopedia Math. Appl. 27, Cambridge Univ. Press, Cambridge, 1989.

[3] H. Bremermann, Distributions, Complex Variables and Fourier Transforms, AddisonWesley, Reading, Massachusetts, 1965.

[4] R. Bojanić and J. Karamata, On a class of functions of regular asymptotic behavior, Math. Res. Centre, U.S. Army, Madison, Wis., Tech. Summary Rep. No. 436, 1963.

[5] Yu. N. Drozhzhinov and B. I. Zav'yalov, Asymptotically homogeneous generalized functions and boundary properties of functions holomorphic in tubular cones, Izv. Math. 70 (2006), 1117-1164.

[6] R. Estrada, Characterization of the Fourier series of distributions having a value at a point, Proc. Amer. Math. Soc. 124 (1996), 1205-1212.

[7] R. Estrada, The Cesàro behaviour of distributions, Proc. R. Soc. Lond. Ser. A Math. Phys. Eng. Sci. 454 (1998), 2425-2443.

[8] R. Estrada, A Distributional Version of the Ferenc-Lukács Theorem, Sarajevo J. Math. 1 (2005), 75-92.

[9] R. Estrada and R. P. Kanwal, A Distributional Approach to Asymptotics: Theory and Applications, 2nd ed., Birkhäuser, Boston, 2002.

[10] S. Łojasiewicz, Sur la valeur et la limite d'une distribution en un point, Studia Math. 16 (1957), 1-36.

[11] S. Pilipović, Some properties of the quasiasymptotic of Schwartz distributions. Part I: Quasiasymptotic at $\pm \infty$, Publ. Inst. Math. (Beograd) (N.S.) 43 (1988), 125-130.

[12] S. Pilipović, Some properties of the quasiasymptotic of Schwartz distributions. Part II: Quasiasymptotic at 0, Publ. Inst. Math. (Beograd) (N.S.) 43 (1988), 131-135.

[13] S. Pilipović, On the quasiasymptotic of Schwartz distributions, Math. Nachr. 137 (1988), $19-25$.

[14] S. Pilipović, On the behavior of a distribution at the origin, Math. Nachr. 141 (1989), $27-32$. 
[15] S. Pilipović and B. Stanković, Structural theorems for the S-asymptotic and quasiasymptotic of distributions, Math. Pannon. 4 (1993), 23-35.

[16] S. Pilipović, B. Stanković and A. Takači, Asymptotic Behaviour and Stieltjes Transformation of Distributions, Teubner-Texte zur Mathematik, Leipzig, 1990.

[17] L. Schwartz, Théorie des Distributions, Hermann, Paris, 1966.

[18] E. Seneta, Regularly Varying Functions, Lecture Notes in Mathematics, 508, SpringerVerlag, Berlin-New York, 1976.

[19] B. Stanković, Structural problems of the asymptotic behaviour of generalized functions, in: Generalized Functions and Their Applications (Varanasi, 1991), Plenum, New York, 1993, 251-263.

[20] J. Vindas, Structural theorems for quasiasymptotics of distributions at infinity, Pub. Inst. Math. (Beograd) (N.S) 84(98) (2008), 159-174.

[21] J. Vindas and R. Estrada, Distributionally regulated functions, Studia Math. 181 (2007), $211-236$.

[22] J. Vindas and R. Estrada, Distributional point values and convergence of Fourier series and integrals, J. Fourier Anal. Appl. 13 (2007), 551-576.

[23] J. Vindas and S. Pilipović, Structural theorems for quasiasymptotics of distributions at the origin, Math. Nachr. 282 (2009), 1584-1599.

[24] V. S. Vladimirov, Yu. N. Drozhzhinov and B. I. Zavialov, Tauberian Theorems for Generalized Functions, Kluwer Academic Publishers, Dordrecht, 1988.

[25] V. S. Vladimirov, Yu. N. Drozhzhinov and B. I. Zavialov, Tauberian theorems for generalized functions in a scale of regularly varying functions and functionals, Publ. Inst. Math. (Beograd) (N.S.) 71 (2002), 123-132 (in Russian).

[26] G. Walter, Pointwise convergence of distribution expansions, Studia Math. 26 (1966), 143-154.

[27] G. Walter, Fourier series and analytic representation of distributions, SIAM Rev. 12 (1970), 272-276.

[28] P. Wagner, On the quasi-asymptotic expansion of the causal fundamental solution of hyperbolic operators and systems, Z. Anal. Anwendungen 10 (1991), 159-167.

[29] B. I. Zav'yalov, Automodel asymptotic of electromagnetic form factors and the behavior of their Fourier transforms, Teoret. Mat. Fiz. 17 (1973), 178-188 (in Russian).

[30] B. I. Zav'yalov, Asymptotic properties of functions that are holomorphic in tubular cones, Math. USSR-Sb. 64 (1989), 97-113. 